\title{
Évaluation du risque de dysǵlycémie dans la région de Kitikmeot (Nunavut) au moyen de l'outil CANRISK
}

\author{
Ying Jiang, M.D. (1); Susan Rogers Van Katwyk, B. Sc. (1,2); Yang Mao, Ph. D. (1); Heather Orpana, Ph. D. (1,2); \\ Gina Agarwal, M.B.B.S., Ph. D. (3); Margaret de Groh, Ph. D. (1); Monique Skinner, B.A. (4); Robyn Clarke, B. Sc. (4); \\ Howard Morrison, Ph. D. (1)
}

Cet article a fait l'objet d'une évaluation par les pairs.

Diffuser cet article sur Twitter

\section{Résumé}

Introduction : L’Agence de la santé publique du Canada a adapté un outil finlandais de dépistage du diabète (FINDRISC) dans le but de créer un outil, CANRISK, conçu sur mesure pour la population multiethnique du Canada. Cet outil a été élaboré à partir de données recueillies dans sept provinces canadiennes. Dans le but d'élargir son applicabilité aux populations des territoires nordiques, nous avons réalisé une étude auprès de la population majoritairement inuite de la région de Kitikmeot (Nunavut).

Méthodologie : Nous avons recueilli des questionnaires CANRISK, des mesures physiques et des échantillons de sang auprès de participants vivant dans cinq collectivités de la région de Kitikmeot. Nous avons utilisé une régression logistique pour évaluer l'ajustement du modèle à l'aide des facteurs de risque de l'outil CANRISK d'origine associés à la dysglycémie (prédiabète et diabète). La dysglycémie a été évaluée au moyen d'un test de glycémie plasmatique à jeun (GPJ) administré seul ou avec une épreuve d'hyperglycémie provoquée par voie orale. Nous avons mis au point les scores CANRISK des participants pour tester le fonctionnement de cet outil au sein d'une population inuite.

Résultats : Au total, 303 personnes ont participé à l'étude. La moitié d'entre elles avaient moins de 45 ans, les deux tiers étaient des femmes et $84 \%$ étaient inuites. Sur les 303 participants, $18 \%$ étaient atteints de prédiabète et $4 \%$ avaient un diabète non diagnostiqué. La probabilité de présenter un état dysglycémique augmentait de façon exponentielle avec l'âge et la relation avec l'indice de masse corporelle (IMC) suivait une courbe en U. Par rapport aux résultats d'analyse en laboratoire, l'outil CANRISK avec un seuil de 32 points a présenté une sensibilité de $61 \%$, une spécificité de $66 \%$, une valeur prédictive positive de $34 \%$ et un taux d'exactitude de $65 \%$.

Conclusion : L'outil CANRISK a permis de détecter la dysglycémie au sein de cette population très majoritairement inuite avec une exactitude comparable à celle obtenue avec un échantillon de Canadiens présentant une diversité multiethnique. L'outil CANRISK est donc utilisable pour la région de Kitikmeot, et plus généralement au Nunavut.

Mots-clés : CANRISK, prédiabète, diabète, dysglycémie, Nunavut, Inuits

\section{Introduction}

Le diabète (de type 1 et de type 2 ) et ses complications imposent un lourd fardeau aux Canadiens et au système de santé ${ }^{1}$. De récentes données indiquent que $10 \%$ des
Canadiens âgés de 20 ans et plus ont reçu un diagnostic de diabète ${ }^{2}$. En outre, on estime qu'au moins une personne diabétique sur cinq n'a jamais été diagnostiquée et ignore qu'elle est atteinte de cette maladie ${ }^{3}$.

\section{Points saillants}

- Le questionnaire CANRISK est un outil qui permet d'évaluer le risque de dysglycémie dans la population multiethnique du Canada.

- D’après notre étude au sein d'un échantillon du Nunavut, $18 \%$ des participants étaient atteints de prédiabète et $4 \%$ présentaient un diabète non diagnostiqué.

- L'outil CANRISK appliqué à un échantillon de population très majoritairement inuite a permis une exactitude comparable à celle obtenue avec un échantillon de Canadiens présentant une diversité multiethnique.

- L'outil CANRISK est utilisable dans la région de Kitikmeot, et plus généralement au Nunavut.

Parmi les facteurs de risque favorisant le développement d'un diabète de type 2, mentionnons l'adiposité, l'âge, une prédisposition génétique, des facteurs épigénétiques, le sexe masculin, une mauvaise alimentation, l'inactivité physique et d'autres affections concomitantes comme l'hypertension $^{4,5}$. Des mesures génétiques de substitution intègrent les antécédents familiaux de diabète et l'origine ethnique, les personnes d'origine sud-asiatique ${ }^{6}$ ou issues des Premières Nations ${ }^{3}$ présentant un risque particulièrement élevé d'être atteintes d'un diabète de type 2 .

Rattachement des auteurs :

1. Agence de la santé publique du Canada, Ottawa (Ontario), Canada

2. Université d'Ottawa, Ottawa (Ontario), Canada

3. Département de médecine familiale, Université McMaster, Hamilton (Ontario), Canada

4. Kitikmeot Region Health and Social Services, Cambridge Bay (Nunavut), Canada

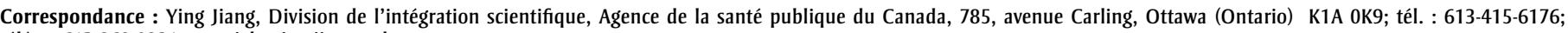
téléc. : 613-960-0921; courriel : ying.jiang@phac-aspc.gc.ca 
En 1987, un examen exhaustif des dossiers médicaux d'Inuits canadiens n'a permis de relever que 31 cas prévalents de diabète chez des adultes de 25 ans et plus, pour une prévalence normalisée selon l'âge de 0,6 \% ${ }^{7}$. Dans l'Enquête sur la santé des Inuits adultes de 2007-2008 menée lors de l'Année polaire internationale ${ }^{8}$, on a constaté une prévalence du diabète de $5,1 \%$ en utilisant l'épreuve d'hyperglycémie provoquée par voie orale (HGPO), soit une prévalence comparable à celle de l'ensemble de la population canadienne, mais des taux élevés d'obésité chez les Inuits par rapport à l'ensemble de la population canadienne'. En raison de l'importance de l'obésité dans l'étiologie du diabète de type 2 , il y a lieu de croire que le taux de diabète de type 2 augmentera probablement au sein de cette population dans un avenir rapproché.

Il est important d'une part de repérer les personnes susceptibles de développer un diabète de type 2 et de les encourager à apporter des changements sains à leur mode de vie pour prévenir cette maladie ou au moins en retarder l'apparition et d'autre part de repérer les cas qui n'ont jamais été diagnostiqués afin de pouvoir amorcer un traitement.

Des analyses sanguines sont nécessaires pour déterminer l'état prédiabétique ou diabétique d'une personne. Si celles-ci sont effectuées sur l'ensemble de la population (p. ex. un dépistage aux trois ans pour les Canadiens âgés de 40 ans et plus, comme le recommandent les lignes directrices publiées en 2013 par l'Association canadienne du diabète ${ }^{10}$ ), il en résulte le dépistage d'un grand nombre de personnes à faible risque. Il est plus économique de repérer les personnes à haut risque grâce à une présélection initiale par questionnaire et d'effectuer ensuite des analyses sanguines poussées chez ces personnes uniquement. C'est l'approche utilisée par la Finlande avec l'élaboration du questionnaire (FINDRISC) ${ }^{11}$, fondé sur les facteurs de risque connus du diabète de type 2 . Au Canada, le questionnaire FINDRISC a été modifié à partir des recommandations d'un comité consultatif d'experts pour tenir compte de la composition multi-ethnique du Canada et d'autres facteurs de risque absents dans le questionnaire FINDRISC mais considérés comme pertinents en contexte canadien $^{12,13}$. Ce questionnaire, CANRISK, a été testé de 2007 à 2011 auprès d'un échantillon de commodité composé d'adultes principalement âgés de 40 à 78 ans provenant de sept provinces, en parallèle avec un test de glycémie plasmatique à jeun (GPJ) et une épreuve d'hyperglycémie provoquée par voie orale (HGPO $)^{12,13}$. Ces données ont été utilisées pour créer et tester le modèle CANRISK grâce à un échantillon d'étalonnage et de validation. Le modèle CANRISK a été comparé au modèle FINDRISC et à un modèle intégrant seulement l'indice de masse corporelle (IMC), le tour de taille, le sexe et l'âge. On a choisi le modèle maximisant la classification des résultats réellement positifs et on l'a évalué pour l'ajuster et pour mesurer la signification statistique de chaque prédicteur grâce à un échantillon d'étalonnage. On a ensuite effectué une validation croisée du modèle final avec un échantillon indépendant ${ }^{12,14,15}$. Des validations de l'utilisation de l'outil de cotation CANRISK chez les jeunes adultes (20 à 39 ans) au sein de certaines populations ethniques sont en cours. L'outil CANRISK est utilisé dans plusieurs régions du Canada, en particulier au Nouveau-Brunswick ${ }^{16}$ et à Toronto, et par des organismes comme Shoppers Drug Mart (Pharmaprix) ${ }^{17}$ et l'Association canadienne du diabète ${ }^{18}$.

D’autres pays ont élaboré des outils d'évaluation semblables à FINDRISC et à CANRISK, par exemple l'outil d'évaluation du risque de diabète de type 2 de l'Australie (AUSDRISK) ${ }^{19}$, l'outil d'évaluation du score de risque de diabète du Royaume-Uni ${ }^{20}$ et l'outil d'évaluation du risque de diabète de type 2 des États-Unis ${ }^{21}$. L'une des principales différences entre ces outils est l'inclusion, au cours de leurs étapes de développement, de groupes ethniques différents, spécifiques à chaque pays (p. ex. dans le cas de l'outil AUSDRISK, les Aborigènes et les peuples insulaires du détroit de Torres). Ainsi, même si la version actuelle de CANRISK a été mise au point sur la base d'un échantillon composé de nombreux groupes ethniques dont les Premières Nations, elle n'a pas été validée avec la population inuite. Notre étude visait à examiner l'ajustement du modèle CANRISK au sein d'une population majoritairement inuite et en testant la sensibilité et la spécificité de l'outil CANRISK ainsi que ses valeurs seuil pour détecter la dysglycémie (prédiabète et diabète).

\section{Méthodologie}

L'Agence de la santé publique du Canada (ASPC) a mené, en collaboration avec le ministère de la Santé et des Services sociaux du Nunavut et les autorités communautaires de santé publique, une étude de cotation du risque de dysglycémie à l'intention de la population du Nunavut, conformément aux lignes directrices de la deuxième édition de l'Énoncé de politique des trois Conseils ${ }^{22}$. La mobilisation communautaire s'est faite par l'entremise de la Kitikmeot Inuit Association (Association inuite de Kitikmeot). L'étude a reçu l'approbation du Comité d'éthique de la recherche de Santé Canada/ASPC et l'autorisation de l'Institut de recherche du Nunavut.

\section{Population étudiée et recrutement}

Entre janvier 2013 et mars 2013, nous avons contacté des résidents âgés de 30 ans et plus des cinq collectivités du Nunavut de la région de Kitikmeot, soit Cambridge Bay, Gjoa Haven, Kugaaruk, Kugluktuk et Taloyoak, pour les inviter à participer à une étude d'évaluation du risque de dysglycémie. Nous avons exclu de l'étude les personnes dont l'état diabétique était connu afin que la méthodologie corresponde à celle utilisée dans l'étude CANRISK d'origine. Pour faire connaître le projet, nous avons eu recours à des annonces radiophoniques, à des avis sur les médias sociaux comme Facebook et à des affiches dans les collectivités. Toutes les personnes ayant fréquenté les centres de santé communautaire ont été appelées à participer au projet et à transmettre le message à leurs amis et aux membres de leur famille. Un bon alimentaire d'une valeur de 50 \$ a été remis aux participants ayant fourni tous les renseignements requis (questionnaire et analyses sanguines).

\section{Collecte des données}

Nous avons convoqué à l'unité locale de santé publique les personnes ayant donné leur consentement éclairé pour passer une entrevue personnelle, se soumettre à des mesures anthropométriques et fournir deux échantillons de sang. Les participants à l'étude ont été pesés à l'aide d'un pèsepersonne numérique (vêtus de vêtements d'intérieur et sans chaussures) et leur taille a été déterminée au moyen d'un ruban à mesurer normalisé fixé au mur. La taille a été mesurée au centimètre près et le poids aux 100 grammes près. Le tour de taille a été mesuré à la circonférence minimale entre le nombril et le processus xiphoïde, au demicentimètre près. Les travailleurs de la santé des diverses collectivités ont pu au besoin apporter leur aide aux participants en anglais, en inuinnaqtun et en inuktitut, et ils 
ont reçu une formation normalisée sur la façon d'effectuer les mesures anthropométriques afin qu'elles soient uniformisées pour les cinq collectivités à l'étude.

Le questionnaire CANRISK ${ }^{16}$ comporte des questions sur le sexe, l'âge, l'activité physique, la consommation de fruits et de légumes, les antécédents d'hypertension, les antécédents d'hyperglycémie, les antécédents familiaux de diabète, l'origine ethnique et la scolarité. Il faut en moyenne moins de cinq minutes pour le remplir. $\mathrm{Au}$ Nunavut, pour tenir compte de l'intérêt manifesté par les communautés locales, ces entrevues ont été complétées par des questions d'ordre alimentaire sur la consommation de "malbouffe » (ce qui inclut les boissons sucrées ou édulcorées) et les " aliments traditionnels ». La " malbouffe» englobait les croustilles, les grignotines, les bâtonnets au fromage, les boissons sucrées de types boissons gazeuses, boissons sucrées en poudre (p. ex. boissons aux fruits/boissons pour sportifs, thé glacé, chocolat chaud) et barbotines. Les " aliments traditionnels » comprenaient le muktuk, le caribou, le bœuf musqué, les oiseaux, les foies d'animaux, la viande de mammifère marin comme le phoque annelé ou la baleine, les poissons comme l'omble chevalier ou les poissons à chair blanche.

Nous avons demandé aux participants de fournir deux échantillons de sang veineux, l'un prélevé à jeun et l'autre prélevé deux heures après la prise d'une boisson contenant $75 \mathrm{~g}$ de glucose (épreuve HGPO), comme le recommandent l'Organisation mondiale de la santé (OMS) et les lignes directrices de 2013 de l'Association canadienne du diabète ${ }^{10,23}$. La mesure du glucose plasmatique à jeun et par l'épreuve HGPO a été effectuée pour tous les échantillons sanguins par le laboratoire DynaLIFE ${ }_{\mathrm{Dx}}$ à Edmonton.

\section{Analyse des données}

Nous avons calculé, pour chacune des collectivités et pour l'ensemble de l'échantillon, le taux de participation à l'étude en comparant les caractéristiques de l'échantillon aux données de population tirées du recensement de 2011.

Nous avons traité l'âge, l'IMC $\left(\mathrm{kg} / \mathrm{m}^{2}\right)$ et le tour de taille comme des variables nominales, en utilisant les catégories du questionnaire CANRISK ${ }^{13}$. Les catégories d'âge étaient : 30 à 44 ans, 45 à 54 ans, 55 à
64 ans et 65 ans et plus. Les catégories d'IMC étaient : IMC inférieur à 25 , IMC compris entre 25 et 29,9 , IMC compris entre 30 et 34,9 et IMC de 35 et plus ${ }^{24}$. Les catégories pour le tour de taille étaient : petit (moins de $94 \mathrm{~cm}$ pour les hommes et de $80 \mathrm{~cm}$ pour les femmes), moyen (de 94 à $102 \mathrm{~cm}$ pour les hommes et de 80 à $88 \mathrm{~cm}$ pour les femmes) et grand (plus de $102 \mathrm{~cm}$ pour les hommes et plus de $88 \mathrm{~cm}$ pour les femmes) ${ }^{25}$. Nous avons mesuré les antécédents familiaux de diabète en calculant le nombre de catégories de parents au premier degré touchés par le diabète (entre 0 et 4). Les niveaux de scolarité étaient : études collégiales ou universitaires partielles, diplôme d'études secondaires et sans diplôme d'études secondaires.

D’autres variables étaient binaires, c'est-àdire qu'il fallait répondre " oui » ou " non » à des questions telles que : "Faites-vous habituellement de l'activité physique, comme de la marche rapide, au moins 30 minutes par jour? »; "Vous a-t-on déjà dit que vous faisiez de l'hypertension artérielle? "; "Vous a-t-on déjà dit que vous faisiez de l'hyperglycémie? » et « Avez-vous consommé des aliments traditionnels dans la dernière année? » Nous avons évalué la consommation de fruits et de légumes à l'aide de la question " À quelle fréquence mangez-vous des fruits ou des légumes? », dont les catégories de réponse étaient « Tous les jours » ou « Pas tous les jours ».

La dysglycémie a été déterminée à partir des résultats au test de GPJ et à l'épreuve HGPO auxquels ont été soumis les participants, et l'état diabétique et prédiabétique ont été définis conformément aux normes de l'OMS ${ }^{23}$. Une personne a été considérée comme prédiabétique si sa GPJ était comprise entre 6,1 et moins de $7,0 \mathrm{mmol} / \mathrm{L}$ et/ou si sa glycémie se situait entre 7,8 et 11,0 mmol/L à l'épreuve HGPO. Une personne a été considérée comme diabétique si sa GPJ était égale ou supérieure à 7,0 mmol/L et/ou si sa glycémie était égale ou supérieure à $11,1 \mathrm{mmol} / \mathrm{L}$ à l'épreuve HGPO.

Nous avons d'abord effectué une régression logistique binaire, avec les variables $\mathrm{du}$ questionnaire CANRISK et les variables supplémentaires liées à l'alimentation, la variable de résultat étant la présence ou l'absence de dysglycémie. Nous avons effectué une deuxième régression logistique binaire en forçant seulement les variables du questionnaire CANRISK dans le modèle. Nous avons utilisé le test de validité de l'ajustement de HosmerLemeshow pour évaluer l'ajustement du modèle, ainsi que la statistique du pseudoR-carré pour comparer ces modèles. Nous avons calculé les rapports de cotes (RC) et les intervalles de confiance (IC) à $95 \%$. Nous avons utilisé l'aire sous la courbe (ASC) de la courbe caractéristique de la performance d'un test (ROC) pour évaluer la capacité de prédiction des modèles. Les scores varient de 0,5 (aucune capacité de prédiction) à 1,0 (capacité de prédiction parfaite). Toutes les analyses ont été réalisées au moyen du logiciel SAS 9.3 (SAS Institute Inc., Cary, Caroline du Nord, États-Unis).

Nous avons ensuite attribué des scores CANRISK aux participants en utilisant l'outil CANRISK accessible au public, et nous avons classé ces scores comme positifs ou négatifs en référence à diverses valeurs seuil : légèrement élevé $(\geq 21)$, modéré $(\geq 29)$, équilibré $(\geq 32)$ et élevé $(\geq 33)^{12}$. Un score égal ou supérieur au seuil a été codé comme positif et un score inférieur au seuil comme négatif. La sensibilité est la proportion d'individus ayant un score CANRISK positif parmi ceux ayant obtenu un résultat positif au test de GPJ et/ou à l'épreuve HGPO. La spécificité est la proportion des individus ayant un score CANRISK négatif parmi ceux ayant obtenu un résultat négatif au test de GPJ et à l'épreuve HGPO. La valeur prédictive positive est la probabilité que les sujets ayant un score de risque CANRISK positif aient un résultat positif au test de GPJ et/ou à l'épreuve HGPO. Le taux d'exactitude est le nombre de scores CANRISK positifs confirmés par des résultats positifs au test de GPJ et/ou à l'épreuve HGPO et le nombre de scores CANRISK négatifs confirmés par des résultats négatifs au test de GPJ et à l'épreuve HGPO par rapport au nombre total de participants. L'objectif de ces statistiques était dans cette étude de déterminer si l'outil CANRISK et les seuils actuels étaient applicables à la population inuite.

\section{Résultats}

Nous avons recueilli au total 303 questionnaires CANRISK et dossiers d'épreuves HGPO valides provenant des cinq collectivités choisies, soit approximativement $11,6 \%(303 / 2614)$ de la population locale âgée de 30 ans ou plus (tableau 1). Environ la moitié des participants étaient âgés de 
TABLEAU 1

Taux de participation à l'étude CANRISK dans la région de Kitikmeot (Nunavut), par collectivité, Canada, 2013

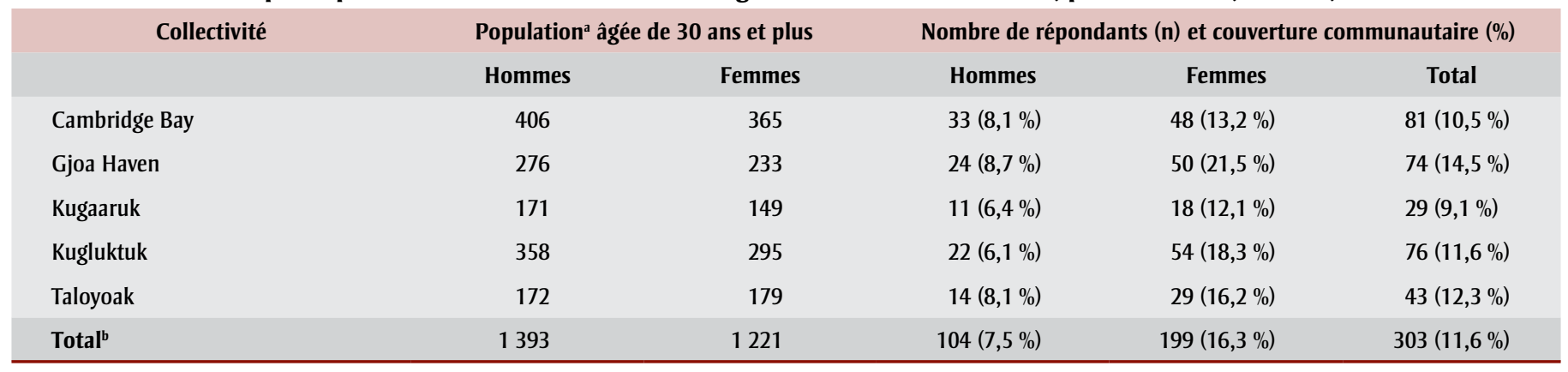

${ }^{\text {a }}$ Les estimations de la population sont fondées sur les données du recensement de 2011 corrigées pour tenir compte du sous-dénombrement net du recensement.

${ }^{\mathrm{b}}$ Les totaux de la région de Kitikmeot comprennent les territoires non organisés et les établissements éloignés.

30 à 45 ans, les deux tiers étaient des femmes et $84 \%$ étaient inuits.

Le tableau 2 montre les principales caractéristiques de risque des participants à l'étude. Parmi les personnes sondées, $67 \%$ étaient en surpoids ou obèses et $56 \%$ présentaient un tour de taille à risque élevé (soit supérieur à $88 \mathrm{~cm}$ pour les femmes et à $102 \mathrm{~cm}$ pour les hommes). Le score CANRISK moyen pour cet échantillon $(\mathrm{N}=303$ ) était de 29,0 (écart-type $=12$, médiane $=28$, minimum $=0$ et maximum $=65)$.

D’après les analyses de laboratoire, 18 \% des participants à l'étude souffraient de prédiabète et $4 \%$ de diabète. La proportion de l'échantillon de personnes considérées comme prédiabétiques variait, selon les collectivités, de 12 à $22 \%$ (tableau 3).

Le tableau 4 présente les résultats des analyses de régression logistique. L'âge et la macrosomie (le fait de donner naissance à un bébé de 4,1 kg [9 lb] ou plus) étaient des prédicteurs significatifs de dysglycémie dans les modèles ajustés complets. Les probabilités de dysglycémie augmentaient avec l'âge, même si les estimations ne se sont pas révélées toutes significatives sur le plan statistique et les probabilités de dysglycémie en fonction de l'IMC et du tour de taille semblaient suivre une courbe en U. Dans le modèle 1, qui incluait les variables supplémentaires liées à l'alimentation, le test de validité de l'ajustement de Hosmer-Lemeshow pour le modèle complet était $p=0,35$, le pseudo-R-carré remis à l'échelle maximale était de 0,23 et l'ASC était de 0,75 . Pour le modèle 2 , soit le modèle CANRISK sans les variables supplémentaires, le test de validité de l'ajustement de Hosmer-Lemeshow était $p=0,94$, le pseudo-R-carré remis à l'échelle maximale de 0,22 et l'ASC de 0,75. Le pseudo-Rcarré remis à l'échelle maximale était légèrement meilleur pour le modèle 1 , ce qui est attendu dans un modèle ayant plus de prédicteurs. Le test de validité de l'ajustement de Hosmer-Lemeshow indique que les deux modèles sont bien ajustés aux données. Les deux modèles présentent une exactitude mesurée par ASC comparable.

Nous avons calculé le score CANRISK pour chaque individu. Les calculs de sensibilité et de spécificité fondés sur les seuils de CANRISK $^{12}$ sont présentés dans le tableau 5. Avec le score de CANRISK " élevé » de 33 ou plus comme critère de classification de la dysglycémie, la sensibilité était de $61 \%$ et la spécificité était de $67 \%$, avec le score de CANRISK « légèrement élevé » de 21 ou plus, la sensibilité était de $85 \%$ et la spécificité était de $31 \%$ (tableau 5). Un calcul ultérieur de la puissance a indiqué que l'étude avait une puissance statistique de 0,84 par rapport à une hypothèse nulle du taux de prévalence de la dysglycémie de 0,15 ( $\alpha=0,05)$.

\section{Analyse}

Les estimations de la région du Nunavut de Kitikmeot se sont révélées très similaires à celles recensées dans les centres de collecte de données de sept provinces (ColombieBritannique, Saskatchewan, Manitoba, Ontario, Nouveau-Brunswick, NouvelleÉcosse et Île-du-Prince-Édouard) faisant partie de l'étude CANRISK d'origine ${ }^{12}$ : respectivement $18 \%$ et $16 \%$ pour le prédiabète et $4 \%$ et $5 \%$ pour le diabète non diagnostiqué. Le fait que la population de l'échantillon du Nunavut soit beaucoup plus jeune en moyenne que celle des échantillons des autres centres de collecte de données CANRISK aurait dû donner lieu à un moindre nombre de cas recensés. Cependant, la prévalence du diabète non diagnostiqué dans cet échantillon de commodité correspond assez bien à la prévalence du diabète relevée dans l'Enquête sur la santé des Inuits adultes menée lors de l'Année polaire internationale fondée sur l'épreuve $\mathrm{HGPO}^{8}$.

La prévalence de la dysglycémie a augmenté de façon exponentielle avec l'âge dans la population à l'étude, les probabilités doublant tous les dix ans, tandis qu'une tendance croissante linéaire plus modeste $\mathrm{du}$ rapport de cotes selon l'âge ${ }^{12}$ a été observée pour les données CANRISK d'origine. Il est difficile de savoir si cela est dû au fait que l'échantillon est relativement réduit ou si cela correspond à une différence sous-jacente du risque selon l'âge pour une population très majoritairement inuite.

L'association entre l'IMC et la dysglycémie au Nunavut n'a montré aucune augmentation linéaire, comme pour l'étude CANRISK d'origine $^{12}$ : il semble plutôt y avoir une relation en $U$, quoique non statistiquement significative. Il semble aussi y avoir une relation en U similaire pour le tour de taille, également non statistiquement significative. Ces observations nous indiquent que les seuils d'IMC définissant les catégories de risque accru pour la santé pourraient devoir être ajustés pour les populations inuites. Cette hypothèse est renforcée par les résultats d'une étude menée auprès d'Inuits du Groenland, dans laquelle on a observé que la tendance chez les Inuits à avoir des jambes plus courtes par rapport au torse ferait en sorte que l'on devrait parler de surpoids à partir d'un IMC de 27 plutôt que de 25 (25 étant la norme de 
TABLEAU 2

Caractéristiques du risque des participants à l'étude CANRISK dans la région de Kitikmeot (Nunavut), Canada, 2013

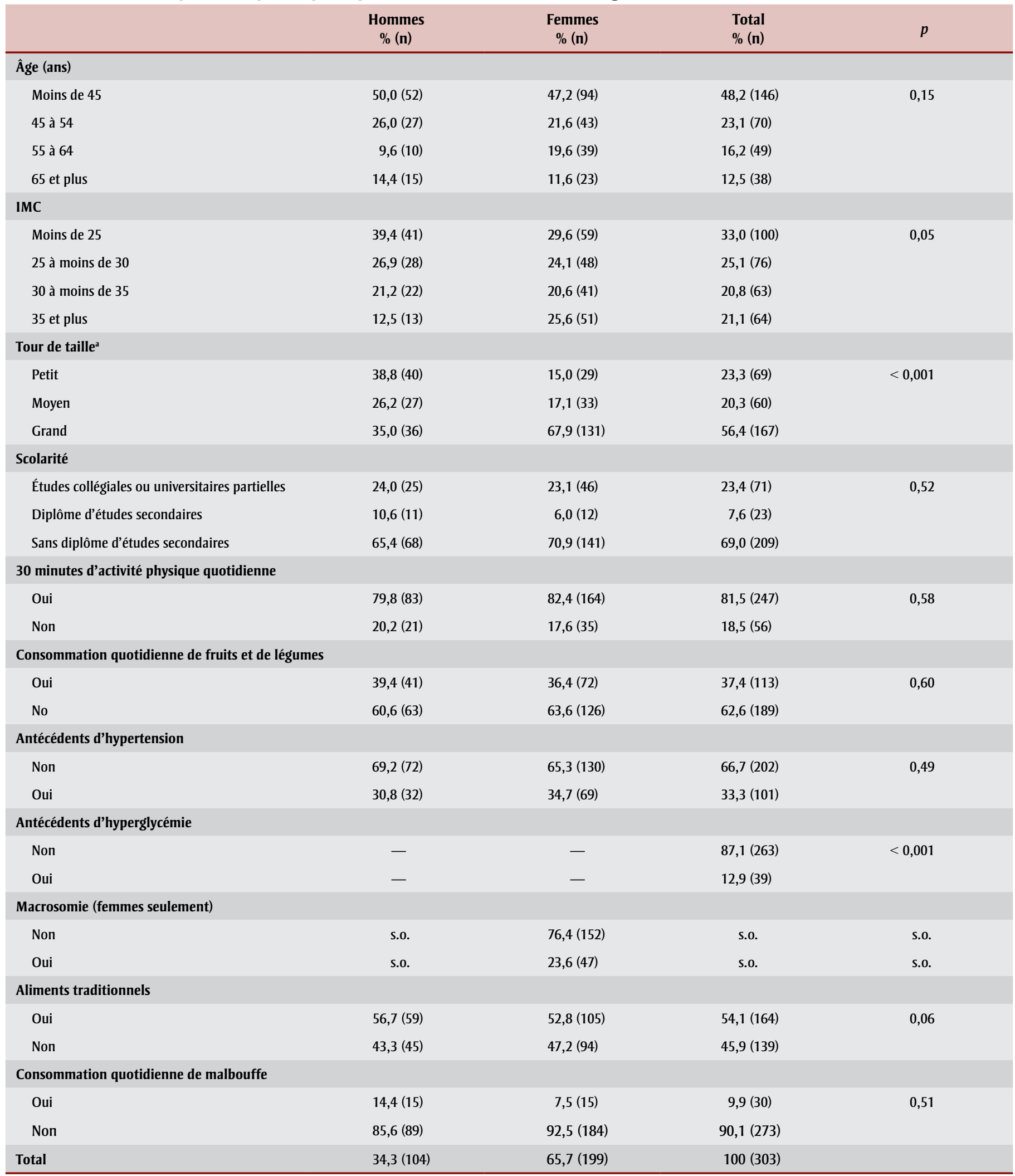

Abréviation : s.o., sans objet.

Remarque : -, données supprimées pour protéger la vie privée $(n<5)$.

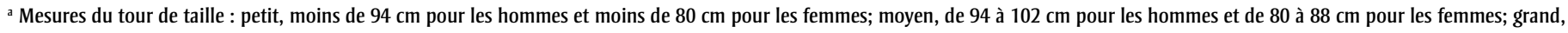
plus de $102 \mathrm{~cm}$ pour les hommes et plus de $88 \mathrm{~cm}$ pour les femmes. 
TABLEAU 3

État dysǵlycémique des participants à l'étude CANRISK dans la région de Kitikmeot (Nunavut), Canada, 2013

\begin{tabular}{llcc}
\multicolumn{1}{c}{ Collectivité } & $\begin{array}{c}\text { Prédiabète } \\
\%(\mathbf{n})\end{array}$ & $\begin{array}{c}\text { Diabète } \\
\%(\mathbf{n})\end{array}$ & $\begin{array}{c}\text { Dysglycémie } \\
\%(\mathbf{n})\end{array}$ \\
\hline Cambridge Bay & $22(18)$ & $9(7)$ & $31(25)$ \\
Gjoa Haven & $12(9)$ & 0 & $12(9)$ \\
Kugaaruk & $21(6)$ & 0 & $21(6)$ \\
Kugluktuk & - & - & $21(16)$ \\
Taloyoak & - & - & $26(11)$ \\
Total & $18(55)$ & $4(12)$ & $22(67)$ \\
\hline
\end{tabular}

Remarque :-, données supprimées pour protéger la vie privée $(\mathrm{n}<5)$.

a Étant donné que les participants dont le diabète avait été diagnostiqué étaient exclus du questionnaire CANRISK, cette colonne ne comprend que les cas de diabète n'ayant jamais été diagnostiqués auparavant.

l'OMS) ${ }^{26}$. Un IMC d'environ 27 chez les Inuits correspond alors au degré de dyslipidémie d'un IMC de 25 chez les nonInuits ${ }^{26}$.

$\mathrm{Au}$ cours des dernières décennies, les aliments traditionnels ont été remplacés dans les collectivités autochtones du Canada par des aliments transformés, qui ont une teneur plus élevée en glucides raffinés, en gras, en sodium et en sucre ${ }^{27,28}$. Ce changement a eu des répercussions sur le développement du diabète et d'autres maladies chroniques $^{27,28}$. Les résultats de notre étude, bien qu'ils ne soient pas statistiquement significatifs, invitent à penser qu'une consommation fréquente d'aliments traditionnels était associée à un risque plus faible de dysglycémie et que la malbouffe était associée positivement à la dysglycémie.

La population inuite du Nunavut est la plus jeune du Canada, l'âge médian étant de 23 ans contre 41 ans pour la population non autochtone au pays ${ }^{29}$. Quoique l'outil CANRISK ait déjà été validé pour un échantillon multi-ethnique, le fait que notre étude ait eu pour but de cibler la population inuite implique que la population de notre échantillon était plus jeune que celle de l'étude CANRISK d'origine. Les deux tiers de nos participants faisaient cependant partie du groupe d'âge des 40 ans et plus.

Lorsque nous avons utilisé les catégories de cotation " équilibré " et "élevé " pour indiquer le risque de dysglycémie des individus, nos résultats se sont révélés similaires à ceux de l'étude CANRISK précédente $^{12}$. Pour la catégorie "élevé " (33 points ou plus), nous avons relevé une sensibilité de $61 \%$ contre $66 \%$ avec le questionnaire CANRISK administré dans les sept provinces, une spécificité de $67 \%$ contre $70 \%$, une valeur prédictive positive de $34 \%$ contre $36 \%$ et un taux d'exactitude global de $65 \%$ contre $64 \%$. Il importe de souligner qu'il n'y a pas de sensibilité ou de spécificité idéale ou recommandée pour un outil visant à repérer les personnes à risque élevé de dysglycémie. Même s’il est souhaitable de disposer d'un outil de dépistage qui soit à la fois très sensible et très spécifique, c'est généralement impossible, car lorsqu'on augmente la sensibilité, on diminue la spécificité, et vice versa. L'équilibre choisi constitue un compromis qui tient compte de l'importance de minimiser à la fois les résultats faussement positifs et les résultats faussement négatifs. Nous avons donc comparé nos taux de sensibilité et de spécificité à ceux mentionnés dans la littérature pour différents outils de dépistage ou pour le même outil mais au sein d'une population différente. Le fait de comparer nos taux de sensibilité et de spécificité aussi à ceux des analyses biochimiques existantes ${ }^{30}$ peut contribuer à mesurer l'utilité de CANRISK comme outil de vérification du risque. De manière générale, les outils de dépistage fondés sur un questionnaire offrent une sensibilité et une spécificité plus faibles que les analyses biochimiques mais ils se révèlent habituellement utiles comme outils d'éducation, en particulier chez les personnes à grand risque de développer un diabète de type 2 , et dans des situations où les avantages de la facilité et du faible coût associés aux questionnaires par rapport aux analyses biochimiques l'emportent sur la perte de sensibilité et de spécificité.

L'épreuve HGPO, qui présente une sensibilité de 90 à $93 \%$ et une spécificité de
$100 \%$ pour repérer les personnes atteintes d'un diabète, constitue donc l'examen de référence ${ }^{31}$. Le test de GPJ $(7,0 \mathrm{mmol} / \mathrm{L}$ ou plus) affiche quant à lui une sensibilité de 40 à $59 \%$ et une spécificité de 96 à $99 \%{ }^{31}$. L'outil FINDRISC, largement utilisé dans les populations européennes et qui, rappelons-le, a servi de modèle à l'élaboration de CANRISK, est l'outil de dépistage du diabète le plus étudié ${ }^{32}$. Un score de FINDRISC égal ou supérieur à 12 correspond à une sensibilité de $78 \%$ et une spécificité de $62 \%$ et une valeur seuil FINDRISC égale ou supérieure à 10 offre une sensibilité de $84 \%$ et une spécificité de $61 \%$. L'outil CANRISK d'origine, dans un échantillon plus large et multi-ethnique de Canadiens et avec un seuil de 32 points ${ }^{12}$, offre une sensibilité de $70 \%$ et une spécificité de $67 \%$. Son rôle a surtout été éducatif et son rôle potentiel dans le dépistage clinique n'a pas été évalué. Compte tenu de tous ces éléments, la capacité de prédiction de l'outil CANRISK au sein de la population du Nunavut semble acceptable par rapport aux autres possibilités offertes (hormis l'épreuve HGPO). Il offre de plus l'avantage d'être un questionnaire auto-administré simple et facile à utiliser.

Tout comme dans l'analyse précédente de CANRISK reposant sur la population des provinces, nous avons choisi d'utiliser le test de GPJ et/ou l'épreuve HGPO comme référence pour déterminer l'état dysglycémique. Ce choix est discutable au sens où les tests ne sont pas entièrement interchangeables et où les groupes visés par chaque test ne se recoupent pas toujours. Par exemple, l'analyse des données de CANRISK pour les sept provinces indique que le test de GPJ n'aurait pas détecté $52 \%$ des cas de diabète et $59 \%$ des cas de prédiabète révélés par l'épreuve $\mathrm{HGPO}^{12}$. Néanmoins, comme nous avons utilisé à la fois le test de GPJ et l'épreuve HGPO, étant donné la sensibilité élevée de cette dernière, il est peu probable que nous n'ayons pas détecté les cas réels. Le choix d'utiliser le test de GPJ et/ou l'épreuve HGPO dans notre analyse a été fait en partie pour assurer une comparabilité avec les données générées par l'analyse précédente de CANRISK effectuée dans les provinces ${ }^{12,13}$. De plus, l'épreuve HGPO demeure pour la plupart des cliniciens l'examen de référence pour le diagnostic du diabète.

\section{Points forts et limites}

En raison des changements dans les modes de vie au cours des dernières décennies, de 
TABLEAU 4

Modèles de régression logistique utilisant CANRISK pour la région de Kitikmeot (Nunavut), Canada, 2013

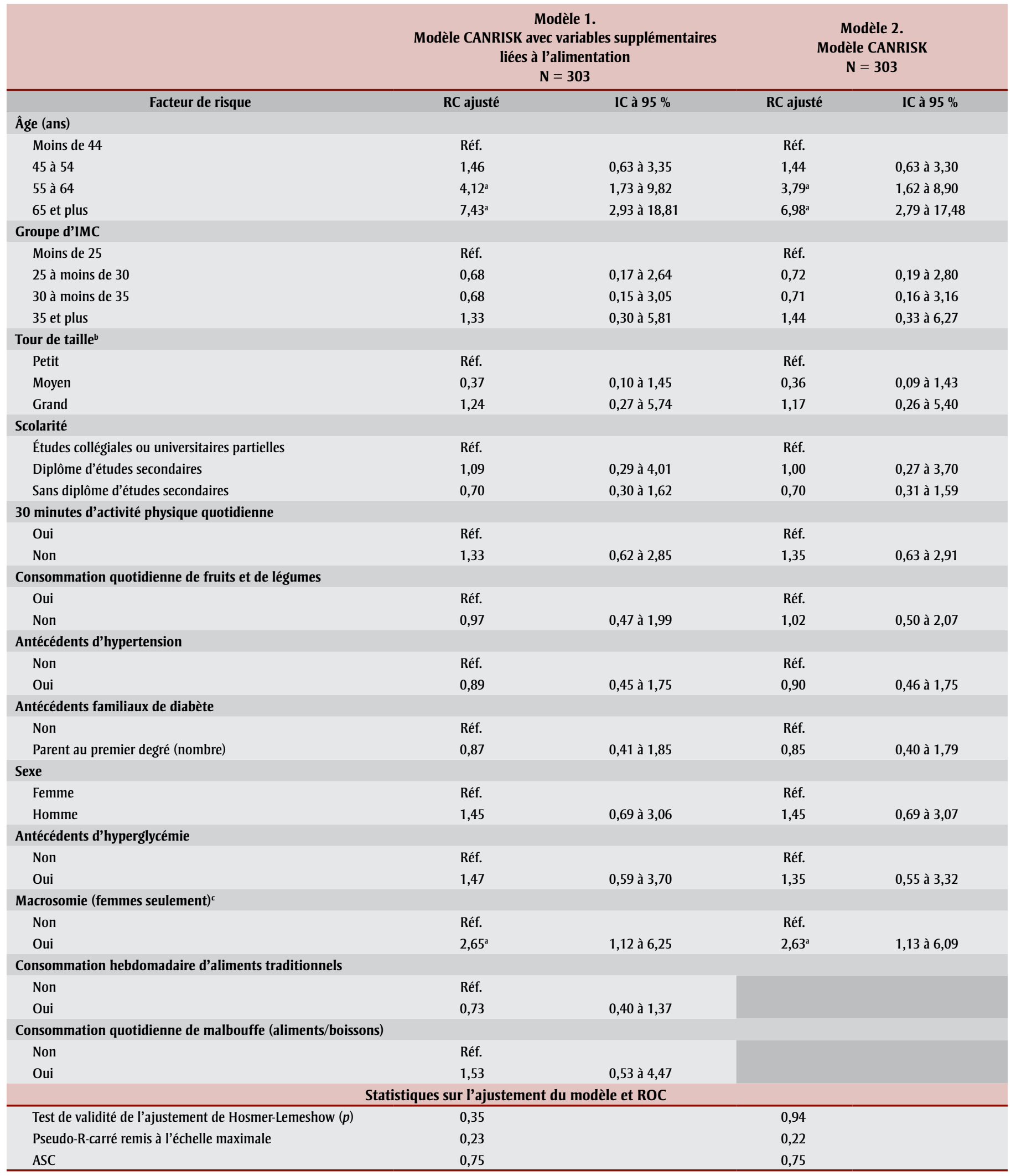

Abréviations : ASC, aire sous la courbe; IC, intervalle de confiance; RC, rapport de cotes; Réf., groupe de référence; ROC, courbe caractéristique de la performance d'un test.

${ }^{\mathrm{a}}$ Indique une signification statistique à $\mathrm{p}<0,05$.

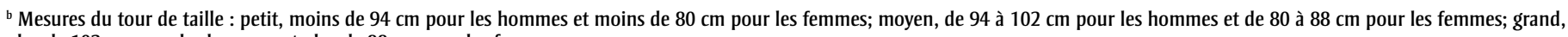
plus de $102 \mathrm{~cm}$ pour les hommes et plus de $88 \mathrm{~cm}$ pour les femmes.

' L'estimation de la macrosomie a été calculée dans le même modèle que les autres paramètres. Les hommes ont constitué une troisième catégorie distincte du groupe de référence. 
TABLEAU 5

Validation de l'outil CANRISK et des valeurs seuil pour la région de Kitikmeot (Nunavut), Canada, 2013

\begin{tabular}{|c|c|c|c|c|}
\hline \multirow{2}{*}{ Analyse de validation } & \multicolumn{4}{|c|}{ CANRISK score } \\
\hline & Légèrement élevé $\geq 21$ & Modéré $\geq 29$ & Équilibréa $^{2} 32$ & Élevé $\geq 33$ \\
\hline Sensibilité & $85,1 \%$ & $65,7 \%$ & $61,2 \%$ & $61,2 \%$ \\
\hline Valeur prédictive positive & $26,0 \%$ & $29,5 \%$ & $33,6 \%$ & $34,2 \%$ \\
\hline Taux d'exactitude global & $43,2 \%$ & $57,8 \%$ & $64,7 \%$ & $65,3 \%$ \\
\hline
\end{tabular}

a Un score équilibré CANRISK est un score optimal visant à établir un équilibre entre la sensibilité et la spécificité du test.

plus amples données probantes sur la prévention et la détection précoce du diabète chez les populations inuites sont nécessaires. Dans ce contexte, notre étude fournit de nouvelles données sur la façon dont l'outil CANRISK est applicable à la population inuite.

Notre étude comporte plusieurs limites. Les femmes étaient surreprésentées dans notre échantillon, même avec une répartition selon l'âge comparable à celle du recensement de 2011 correspondant $^{33}$. L'effet d'un suréchantillonnage des femmes est important seulement en cas d'interactions significatives entre facteurs de risque du modèle et dysglycémie. Notons qu'à l'exception de ce suréchantillonnage des femmes, et malgré l'utilisation d'un échantillon de commodité, notre étude semble largement représentative de la population étudiée : la proportion de participants sans diplôme d'études secondaires (69 \%) et celle de participants inuits (84\%) étaient comparables aux données du recensement (62\% et $90 \%{ }^{33}$ ).

L'étude reposait sur un échantillon de commodité composé de volontaires, mais nous n'avons pas pu mesurer de manière exhaustive les différences entre les volontaires et le reste de la population. Les taux de diabète et de prédiabète non diagnostiqués chez les volontaires ne sont donc pas nécessairement représentatifs de la région étudiée ou de l'ensemble de la population du Nunavut. En outre, comme nous avons exclu les participants dont le diabète avait été diagnostiqué, nous n'avons pu calculer que les taux de prédiabète et les taux de diabète non diagnostiqué. Nous n'avons pas pu estimer le fardeau total de la dysglycémie dans ces collectivités en raison à la fois des faibles taux de participation et de notre incapacité à déterminer de manière systématique si les participants étaient différents ou non des non-participants. Les limites les plus importantes de cette étude sont sans doute la faible taille de son échantillon et le manque de précision des rapports de cotes qui en découle.

À l'instar d'autres outils de dépistage du diabète, comme AUSDRISK ${ }^{19}$ et FINDRISC ${ }^{11}$, l'outil CANRISK utilise un seul modèle prédictif pour tous les groupes d'âge. Bien que CANRISK ait raisonnablement bien performé pour prédire la dysglycémie au sein de notre population, nous n'avons pu examiner de manière fiable, compte tenu de la faible taille de notre échantillon, la performance de cet outil pour les répondants de moins de 40 ans. En effet, même si l'outil CANRISK a été élaboré à partir d'un échantillon incluant des personnes âgées de 18 ans et plus, on ne le recommande actuellement que pour les personnes de 40 ans ou plus. Cette limite se révèle particulièrement pertinente pour les populations inuites, la répartition selon l'âge de ces dernières faisant état d'une population beaucoup plus jeune. D'autres recherches vont donc être nécessaires pour évaluer la performance des outils de dépistage du risque de diabète au sein de populations plus jeunes.

\section{Conclusion}

Notre modèle de régression logistique a fait état d'un ajustement acceptable du modèle lorsque nous avons utilisé les variables du questionnaire CANRISK pour prédire la dysglycémie. De plus, les catégories de risque fondées sur les scores de l'outil CANRISK d'origine ont permis de détecter, dans cet échantillon composé majoritairement d'Inuits, une vraie dysglycémie avec une exactitude comparable à celle obtenue dans l'échantillon multi-ethnique du Canada. L'outil CANRISK se révèle applicable à la région de Kitikmeot et, par conséquent, il pourrait être utilisé de manière similaire pour l'ensemble du Nunavut. Grâce à l'outil CANRISK qui permet de déterminer les niveaux de risque de dysglycémie, les individus, les collectivités et les autorités sanitaires locales peuvent prendre les mesures appropriées afin de réduire le fardeau de la dysglycémie au sein de la population inuite.

\section{Remerciements}

Nous remercions le ministère de Santé du Nunavut, la Kitikmeot Inuit Association et cinq communautés de la région de Kitikmeot, Nunavut (Cambridge Bay, Gjoa Haven, Kugaaruk, Kugluktuk et Taloyoak) de leur soutien.

\section{Conflits d'intérêts}

Les auteurs n'ont aucun conflit d'intérêts à déclarer.

\section{Contributions des auteurs}

YJ, YM et HM ont considérablement contribué à la conception de l'étude et ont rédigé la première ébauche du manuscrit. YM, SRVK et YJ ont analysé les données. MS et RC ont élaboré des outils et effectué l'acquisition des données. HO, GA, MG, HM, YM, SRVK et YJ ont relu et révisé le manuscrit. Tous les auteurs ont lu et donné leur approbation que la version finale de cet article soit publiée, et étaient d'accord de se porter garants du travail.

\section{Références}

1. Association canadienne du diabète, Diabète Québec. Diabète : Le Canada à l'heure de la remise en question : Tracer une nouvelle voie. Canada : Association canadienne du diabète; 2011. En ligne à : http://www.diabetes.ca/CDA /media/documents/publications-and -newsletters/advocacy-reports/canada -at-the-tipping-point-french.pdf 
2. Gouvernement du Canada. Cadre d'indicateurs des maladies chroniques et des blessures : Statistiques rapides [Internet]. Ottawa (Ont.) : Agence de la santé publique du Canada; 2015 [mise à jour le 8 déc. 2015; consulté le 28 juin 2016]. En ligne à : http:// infobase.phac-aspc.gc.ca/cdiif/index -fr.aspx

3. Agence de la santé publique du Canada. Le diabète au Canada : perspective de santé publique sur les faits et chiffres. Ottawa (Ont.) : Agence de la santé publique du Canada; 2011. [no HP35-25/2011F]

4. International Diabetes Federation. Risk factors [Internet]. Brussels: International Diabetes Federation; 2015 [consulté le 18 avril 2016]. En ligne à : http://www.idf.org/about-diabetes /risk-factors

5. American Diabetes Association. Age, race, gender and family history [Internet]. Arlington (VA): American Diabetes Association; 2014 [mis à jour le 12 févr. 2014; consulté le 20 avril 2016]. En ligne à : http://www.diabetes .org/are-you-at-risk/lower-your-risk /nonmodifiables.html

6. Shah A, Kanaya AM. Diabetes and associated complications in the South Asian population. Curr Cardiol Rep. 2014;16(5):476.

7. Young TK, Schraer CD, Shubnikoff EV, Szathmary EJ, Nikitin YP. Prevalence of diagnosed diabetes in circumpolar indigenous populations. Int J Epidemiol. 1992;21(4):730-736.

8. Egeland GM, Cao Z, Young TK. Hypertriglyceridemic-waist phenotype and glucose intolerance among Canadian Inuit: the International Polar Year Inuit Health Survey for Adults 2007-2008. JAMC. 2011;183(9):E553E558.

9. Statistique Canada. Base de données CANSIM : Tableau 105-0507. Indice de masse corporelle (IMC) mesuré chez les adultes, selon le groupe d'âge et le sexe, population à domicile de 18 ans et plus excluant les femmes enceintes, Canada (sauf les territoires). [Internet]. Ottawa (Ont.) : Statistique Canada; 2009 [consulté le 9 juin 2015]. En ligne à : http://www5.statcan.gc.ca/cansim /a26?id = 1050507\&retrLang = fra\&lang $=$ fra
10. Canadian Diabetes Association Clinical Practice Guidelines Expert Committee, Ekoe JM, Punthakee Z, Ransom T, Prebtani AP, Goldenberg R. Screening for type 1 and type 2 diabetes. Can J Diabetes. 2013;37 Suppl 1:S12-S15.

11. Lindstrom J, Tuomilehto J. The diabetes risk score: a practical tool to predict type 2 diabetes risk. Diabetes Care. 2003;26(3):725-731.

12. Robinson CA, Agarwal G, Nerenberg $K$. Validation du modèle pronostique CANRISK pour l'évaluation du risque de diabète dans la population multiethnique du Canada. Maladies chroniques et blessures au Canada. 2011; 32(1):21-35.

13. Kaczorowski J, Robinson C, Nerenberg K. Development of the CANRISK questionnaire to screen for prediabetes and undiagnosed type 2 diabetes. Can J Diabetes. 2009;33(4):381-385.

14. Altman DG, Vergouwe Y, Royston P, Moons KG. Prognosis and prognostic research: validating a prognostic model. BMJ. 2009;338:b605.

15. Altman DG, Royston P. What do we mean by validating a prognostic model? Stat Med. 2000;19(4):453-473.

16. Nouveau-Brunswick Santé. Questionnaire sur le diabète CANRISK [Internet]. Fredericton (NB) : Nouveau-Brunswick Santé; 2014 [mise à jour le 10 sept. 2014; consulté le 9 juin 2015]. En ligne à : http://www2.gnb.ca/content/gnb /fr/ministeres/sante/patients /SoinsDeSantePrimaires/Strategie -globale-sur-le-diabete-pour-les-Neo -Brunswickois-et-Neo-Brunswickoises -2011-2016/Questionnaire-sur-le -diabete_CANRISK.html

17. Pharmaprix. Êtes-vous à risque? [Internet]. Toronto (Ont.) : Pharmaprix; 2015 [mise à jour le 31 mars 2015; consulté le 9 juin 2015]. En ligne à : http://canrisk.pharmaprix.ca/fr/

18. Canadian Diabetes Association. Take the Test [Internet]. Canada : Canadian Diabetes Association; 2014 [mise à jour le 5 nov. 2014; consulté le 9 juin 2015]. En ligne à : www.diabetes.ca /take-the-test/
19. Chen L, Magliano DJ, Balkau B, et al. AUSDRISK: an Australian Type 2 Diabetes Risk Assessment Tool based on demographic, lifestyle and simple anthropometric measures. Med J Aust 2010;192(4):197-202.

20. Diabetes UK. Diabetes Risk Score Assessment Tool [Internet]. London : Diabetes UK; 2015 [consulté le 9 juil. 2015]. En ligne à : https://www.diabetes .org.uk/Professionals/Diabetes-Risk -Score-assessment-tool/

21. American Diabetes Association. Type 2 Diabetes Risk Test [Internet]. Arlington (VA): American Diabetes Association; 2015 [consulté le 9 juin 2015]. En ligne à : http://www.diabetes .org/are-you-at-risk/diabetes-risk-test/

22. Instituts de recherche en santé du Canada (IRSC), Conseil de recherches en sciences naturelles et en génie du Canada (CRSNGC), Conseil de recherches en sciences humaines du Canada (CRSHC). La recherche visant les Premières nations, les Inuits ou les Métis du Canada. Dans : Énoncé de politique des Trois conseils : Éthique de la recherche avec des êtres humains (chapitre 9, p.121-154). Ottawa (Ont.) : CRSHC, CRSNGC et IRSC; 2010. [no MRd21-18/2010F-PDF]

23. Organisation mondiale de la santé, International Diabetes Federation. Definition and diagnosis of diabetes mellitus and intermediate hyperglycemia: report of a WHO/IDF consultation. Genève : Organisation mondiale de la santé; 2006.

24. Organisation mondiale de la santé. Obésité : prévention et prise en charge de l'épidémie mondiale. Rapport d'une consultation de l'OMS. Genève : Organisation mondiale de la santé; 2000. [Série de Rapports techniques 894]

25. Canadian Diabetes Association Clinical Practice Guidelines Expert Committee, Wharton S, Sharma AM, Lau DC. Weight management in diabetes. Can J Diabetes. 2013;37 Suppl 1:S82-S86.

26. Andersen S, Fleischer Rex K, Noahsen P, Sorensen HC, Mulvad G, Laurberg P. Raised BMI cut-off for overweight in Greenland Inuit-a review. Int J Circumpolar Health. 2013;72:21086. doi: 10.3402/ijch.v72i0.21086 
27. Sharma S, Cao X, Roache C, Buchan A, Reid R, Gittelsohn J. Assessing dietary intake in a population undergoing a rapid transition in diet and lifestyle: the Arctic Inuit in Nunavut, Canada. Br J Nutr. 2010;103(5): 749-759.

28. Centre de collaboration nationale de la santé autochtone. Fiche d'information sur la nutrition [Internet]. Prince George (C.-B.) : Centre de collaboration nationale de la Santé autochtone; 2013 [consulté le 9 juin 2015]. En ligne à : http://www.nccah-ccnsa.ca /Publications/Lists/Publications /Attachments/71/Nutrition_FR_web.pdf

29. Statistique Canada. Les peuples autochtones au Canada : Premières Nations, Métis et Inuits : Enquête nationale auprès des ménages, 2011 (Tableau 4) [Internet]. Ottawa (Ont) : Statistique Canada; 2013 [mise à jour le 23 déc. 2015; consulté le 19 juil. 2016]. En ligne à : http://www12.statcan.gc.ca /nhs-enm/2011/as-sa/99-011-x/99-011 -x2011001-fra.cfm

30. Canadian Task Force on Preventive Health Care, Pottie K, Jaramillo A, et al. Recommendations on screening for type 2 diabetes in adults. JAMC. 2012; 184(15):1687-1696.

31. Engelgau MM, Narayan KM, Herman WH. Screening for type 2 diabetes. Diabetes Care. 2000;23(10):1563-1580.

32. Echouffo-Tcheugui JB, Ali MK, Griffin SJ, Narayan KM. Screening for type 2 diabetes and dysglycemia. Epidemiol Rev. 2011;33(1):63-87.

33. Statistique Canada. Kitikmeot, REG, Nunavut (Code 6208) (tableau). Profil de l'Enquête nationale auprès des ménages (ENM). Enquête nationale auprès des ménages (ENM) de 2011 [Internet]. Ottawa (Ont.) : Statistique Canada; 2013 [consulté le 9 juin 2015]. En ligne à : http://www12.statcan.gc .ca/nhs-enm/2011/dp-pd/prof/index .cfm?Lang $=\mathrm{F}$ 